\title{
VIOLÊNCIA FINANCEIRA E SEXUAL CONTRA \\ A PESSOA IDOSA: CARACTERIZAÇÃO DAS NOTIFICAÇÕES NO ESPÍRITO SANTO
}

\author{
FINANCIAL AND SEXUAL VIOLENCE AGAINST \\ THE ELDERLY: CHARACTERIZATION OF \\ NOTIFICATIONS IN ESPÍRITO SANTO
}

\section{VIOLENCIA FINANCIERA Y SEXUAL CONTRA LOS ANCIANOS: CARACTERIZACIÓN DE LAS NOTIFICACIONES EN ESPÍRITO SANTO}

\author{
Franciéle Marabotti Costa Leite \\ Gracielle Pampolim² \\ Mayara Alves Luis ${ }^{3}$ \\ Raniele de Paula Silva ${ }^{3}$ \\ Márcia Regina de Oliveira Pedroso ${ }^{4}$
}

Como citar este artigo: Leite FMC, Pampolim G, Luis MA, Silva RP, Pedroso MRO. Violência financeira e sexual contra a pessoa idosa: caracterização das notificações no Espírito Santo. Rev baiana enferm. 2019;33:e33364.

Objetivo: identificar as prevalências de violência financeira e sexual contra a pessoa idosa notificadas no Espírito Santo entre 2011 e 2018 e descrever as características da vítima, do agressor e da agressão. Método: estudo descritivo, utilizando dados notificados de violência financeira e sexual contra o idoso no Espírito Santo, quanto às características da vítima, do agressor e da agressão. Resultados: a prevalência de violência financeira foi $2,8 \%$ e sexual de 0,7\%. Pessoas idosas do sexo feminino, com baixa escolaridade, de raça preta ou parda e sem deficiência/transtorno representaram os principais casos. As agressões, em sua maioria, foram cometidas por uma única pessoa, do sexo masculino, conhecido da vítima, no domicílio e com histórico de repetição. Conclusão: as violências financeira e sexual notificadas são de baixa prevalência, todavia demonstram a vulnerabilidade do idoso e a importância do profissional de saúde na promoção de um cuidado qualificado pautado na integralidade da assistência.

Descritores: Violência. Idoso. Notificação. Delitos Sexuais. Sistemas de Informação em Saúde.

Objective: to identify the prevalence of financial and sexual violence against the elderly notified in Espirito Santo between 2011 and 2018 and to describe the characteristics of the victim, the offender and the aggression. Method: descriptive study, using reported data on financial and sexual violence against the elderly in Espirito Santo, concerning the characteristics of the victim, the offender and the aggression. Results: the prevalence of financial violence was $2.8 \%$ and sexual violence, $0.7 \%$. Elderly women, with low schooling, black or pardas and without disabilities/disorders represented the major cases. Most aggressions were committed by a single person, male, victim's

\footnotetext{
Enfermeira. Doutora em Epidemiologia. Professora da Universidade Federal do Espírito Santo. Vitória. Espírito Santo, Brasil. https://orcid.org/0000-0002-6171-6972 Fisioterapeuta. Mestre em Políticas Públicas e Desenvolvimento Local. Universidade Federal do Espírito Santo. Professora da Escola Superior da Santa Casa de Misericórdia. Vitória. Espírito Santo, Brasil. graciellepampolim@hotmail.com. https://orcid.org/0000-0002-4I57-352।

Enfermeira. Universidade Federal do Espírito Santo. Vitória. Espírito Santo, Brasil. https://orcid.org/0000-0002-5162-8899; https://orcid.org/0000-0002-0745-050 I

4 Nutricionista. Mestre em Ciências. Universidade Federal do Espírito Santo. Professora da Universidade Federal do Oeste da Bahia. Barreiras, Bahia, Brasil. https://orcid.org/0000-0002-2859-159X
} 
acquaintance, at home and with a bistory of repetition. Conclusion: there is low prevalence of notified financial and sexual violence; however, the study demonstrated the vulnerability of the elderly and the importance of health professionals in promoting a skilled care based on the integrality of care.

Descriptors: Violence. Aged. Notification. Sexual Offenses. Health Information Systems.

Objetivo: identificar la prevalencia de la violencia financiera y sexual contra el anciano notificada en Espírito Santo entre 2011 y 2018, y describir las características de la víctima, del agresor y de la agresión. Método: estudio descriptivo, utilizando datos notificados de la violencia financiera y sexual contra los ancianos en Espirito Santo, como las características de la víctima, del agresor y de la agresión. Resultados: la prevalencia de la violencia sexual fue $2.8 \%$ y de la violencia financiera, 0,7\%. Las mujeres ancianas, con baja escolaridad, negras o pardas y sin discapacidad/trastornos representaron los principales casos. Las agresiones, en su mayorí, fueron cometidas por una sola persona, varón, conocida de la víctima, en casa y con un historial de repetición. Conclusión: la violencia sexual y financiera notificada tiene baja prevalencia, pero muestra la vulnerabilidad de los ancianos y la importancia de los profesionales de la salud para promover una atención especializada basada en la integralidad de la atención.

Descriptores: Violencia. Anciano. Notificación. Delitos Sexuales. Sistemas de Información en Salud.

\section{Introdução}

O envelhecimento populacional é um fenômeno de proporção mundial que, nas últimas décadas, vem ganhando especial destaque nos países em desenvolvimento, sobretudo no Brasil. Neste país, o aumento da expectativa de vida associado à diminuição das taxas de mortalidade, vêm propiciando o incremento do contingente de idosos ${ }^{(1)}$. Esse período de mudança na vida de um indivíduo favorece sua fragilidade e, somado ao preconceito, desrespeito e desigualdades, pode torná-lo mais susceptível a sofrer, por exemplo, situações de violência ${ }^{(2)}$.

A violência contra a pessoa idosa é definida como qualquer ato singular ou repetido, ou a falta de ação devida, que se origine em qualquer relacionamento em que haja expectativa e/ou confiança e resulte em dano físico ou sofrimento psicológico para uma pessoa idosa. É considerada um problema de saúde pública que não distingue classe social e tem sido responsável por diversas consequências negativas à qualidade de vida dos idosos. Pode ser classificada em violência física, psicológica, sexual ou financeira e negligência ${ }^{(3)}$.

Revisões sistemáticas da literatura internacional têm evidenciado que a prevalência de idosos que sofreram algum tipo de violência é de cerca de $15,7 \%{ }^{(4)}$. Quando se analisam os tipos específicos de violência, em especial por região geográfica, esses achados variam consideravelmente, como é o caso das violências financeira e sexual ${ }^{(4-5)}$. Estudos internacionais vêm mostrando que a prevalência de violência financeira é de $4,7 \%$ em média, variando entre 13,1\% na Nigéria a 2,6\% no México, enquanto a prevalência de violência sexual é de $0,7 \%$, com variação de 0,04\% na Nigéria a 1,0\% entre os países europeus ${ }^{(4)}$.

A violência financeira corresponde ao uso ilegal ou inadequado dos recursos financeiros e patrimoniais do idoso. Este tipo de violência é muito comum no meio familiar e, por isso, também é menos denunciado. Já a violência sexual consiste em envolver a pessoa idosa em ato sexual, sem o seu consentimento. Este ato pode ser para excitação, copulação ou outras práticas eróticas $^{(6)}$.

Diante desse cenário, percebe-se que estudar a violência contra a pessoa idosa é de fundamental importância, para que seja possível subsidiar as redes de atenção e acolhimento a essa população e elaborar políticas de enfrentamento a este agravo que sejam resolutivas e eficazes ${ }^{(2)}$. Outro fato digno de nota é que, em razão de suas prevalências consideradas baixas, as violências financeira e sexual são pouco trabalhadas na literatura $^{(7)}$, tanto em âmbito nacional ${ }^{(8)}$ quanto internacional $^{(4-5)}$. É importante destacar que, 
não raramente, esses agravos estão associados também a outros tipos de maus-tratos, que resultam em lesões físicas, psicológicas e até mesmo em óbito ${ }^{(6)}$.

Isso posto e levando-se em consideração a invisibilidade atribuída às violências contra a pessoa idosa e o intuito de contribuir para essa lacuna encontrada na literatura, este estudo objetiva identificar as prevalências de violência financeira e sexual contra a pessoa idosa notificadas no Espírito Santo entre 2011 e 2018 e descrever as características da vítima, do agressor e da agressão.

\section{Método}

Estudo do tipo descritivo, com todos os casos notificados de violência financeira e sexual contra idosos (indivíduos com 60 anos ou mais) atendidos nos serviços de saúde, entre os anos de 2011 e 2018, no estado do Espírito Santo. As informações foram provenientes da base de dados secundários do Sistema de Informação de Agravos e Notificação (SINAN) fornecidos pela Vigilância Epidemiológica da Secretaria do Estado da Saúde (SESA) do Espírito Santo. O ano de 2011 foi escolhido como corte inicial para o estudo em razão de, a partir desse ano, a violência ter passado a integrar a lista de agravos de notificação compulsória, universalizando a notificação desse agravo para todos os serviços de saúde ${ }^{(3)}$.

No SINAN são registrados os dados levantados pela vigilância contínua, que compreende os casos de violência interpessoal e autoprovocada. Este sistema é alimentado pelas informações registradas pelos diversos estabelecimentos conveniados nas fichas de notificações individuais. No caso da violência, essa ficha, conhecida como Ficha de Notificação/Investigação de Violência Interpessoal e Autoprovocada, é subdividida em dez blocos, para melhor agrupamento das informações referentes ao perfil da vítima e do agressor, características da violência e dos encaminhamentos realizados.

Entre março e maio de 2019, foi conduzida uma análise exploratória descritiva para variáveis de interesse e correção dos possíveis erros ou inconsistências do banco, seguindo as diretrizes do Instrutivo de Notificação Interpessoal e Autoprovocada. As notificações de violência contra o idoso foram analisadas segundo os seguintes tipos de violência interpessoal: financeira ( $\operatorname{sim} /$ não) e sexual ( $\operatorname{sim} /$ não); além de características da vítima - faixa etária (60 a 69 anos/70 anos ou mais), sexo (masculino/feminino), raça/cor (branca/ preta-parda), escolaridade (0 a 4 anos/5 anos ou mais), situação conjugal (com companheiro/sem companheiro) e presença de deficiência/transtorno ( $\mathrm{sim} / \mathrm{não}$ ); características do agressor - idade em anos (0 a 59/60 ou mais), sexo (masculino/feminino/ambos), vínculo (conhecidos/desconhecidos) e suspeita de uso de álcool (sim/não); e características da agressão - número de envolvidos (um/dois ou mais), se ocorreu na residência (sim/não), turno (manhã-tarde/noite-madrugada), repetição (sim/não), zona (urbana/rural) e encaminhamentos (sim/não).

Os dados foram processados no programa estatístico Stata versão 13.0 e analisadas por meio da estatística descritiva em frequência bruta e relativa e intervalo de confiança (IC) de 95\%.

O estudo foi aprovado pelo Comitê de Ética em Pesquisa da Universidade Federal do Espírito Santo, sob Parecer número 2.819.597. Foram respeitadas as normas e diretrizes da Resolução n. 499/2012 do Conselho Nacional de Saúde.

\section{Resultados}

No período de 2011 a 2018 foram notificados 1.635 casos de violência contra a pessoa idosa, dos quais 57 foram do tipo financeira ou sexual. Dentre esses, verifica-se que 46 corresponderam à violência financeira, o que equivale a uma prevalência de 2,8\% (IC 95\%: 2,1-3,7), e 11 notificações, isto é, menos de 1\% (P=0,7; IC 95\%: 0,3-1,2), referentes à violência sexual (dados não apresentados em tabela).

A Tabela 1 apresenta a caracterização das notificações de violência financeira e sexual praticadas contra a pessoa idosa, de acordo com os dados da vítima. Quanto aos casos notificados de abuso financeiro, observa-se que a 
maioria foi cometida contra idosos com idade de 70 anos ou mais, do sexo feminino, preta ou parda. Em grande parte das notificações, o idoso tinha quatro anos de escolaridade; a maioria possuía companheiro e não apresentava deficiência/transtorno. No que tange às notificações de abuso sexual, a maior parte das vítimas pertencia à faixa etária de 60 a 69 anos, sendo todas do sexo feminino. Quanto à raça e escolaridade, a maioria era da raça preta ou parda e com escolaridade de até quatro anos de estudo. Em relação à situação conjugal, na maior parte dos casos, a pessoa idosa não apresentava companheiro e grande parte sem manifestar deficiência/transtorno.

Tabela 1 - Caracterização das notificações de violência financeira e sexual praticadas contra a pessoa idosa, de acordo com os dados da vítima. Espírito Santo, Brasil - 2011-2018

\begin{tabular}{|c|c|c|c|c|c|c|}
\hline \multirow{2}{*}{ Variáveis } & \multicolumn{3}{|c|}{$\begin{array}{l}\text { Violência Financeira } \\
\qquad \mathrm{n}=46\end{array}$} & \multicolumn{3}{|c|}{$\begin{array}{c}\text { Violência Sexual } \\
n=11\end{array}$} \\
\hline & $\mathbf{n}$ & $\%$ & $\begin{array}{c}\text { Intervalo de } \\
\text { Confiança } 95 \%\end{array}$ & $\mathbf{n}$ & $\%$ & $\begin{array}{c}\text { Intervalo de } \\
\text { Confiança } 95 \%\end{array}$ \\
\hline \multicolumn{7}{|l|}{ Faixa Etária } \\
\hline 60 a 69 anos & 9 & 19,6 & $10,3-34,0$ & 7 & 63,6 & $31,9-86,7$ \\
\hline 70 ou mais & 37 & 80,4 & $65,9-89,7$ & 4 & 36,4 & $13,3-68,1$ \\
\hline \multicolumn{7}{|l|}{ Sexo } \\
\hline Masculino & 15 & 32,6 & $20,4-47,8$ & - & - & - \\
\hline Feminino & 31 & 67,4 & $52,2-79,6$ & 11 & 100,0 & \\
\hline \multicolumn{7}{|l|}{ Raça/Cor } \\
\hline Branca & 20 & 48,8 & $33,5-64,2$ & 3 & 27,3 & $8,3-60,9$ \\
\hline Preta/Parda & 21 & 51,2 & $35,7-66,4$ & 8 & 72,7 & $39,1-91,7$ \\
\hline \multicolumn{7}{|l|}{ Escolaridade (anos) } \\
\hline 0 a 4 anos & 15 & 55,6 & $35,9-73,6$ & 6 & 66,7 & $30,4-90,2$ \\
\hline 5 anos ou mais & 12 & 44,4 & $26,4-64,1$ & 3 & 33,3 & $9,8-69,6$ \\
\hline \multicolumn{7}{|l|}{ Situação Conjugal } \\
\hline Com companheiro & 35 & 81,4 & $66,4-90,6$ & 4 & 36,4 & $13,3-68,1$ \\
\hline Sem companheiro & 8 & 18,6 & $9,3-33,6$ & 7 & 63,6 & $31,9-86,7$ \\
\hline \multicolumn{7}{|l|}{ Deficiência/Transtorno } \\
\hline $\operatorname{Sim}$ & 10 & 23,8 & $13,0-39,5$ & 2 & 18,2 & $4,1-53,5$ \\
\hline Não & 32 & 76,2 & $60,5-87,0$ & 9 & 81,8 & $46,5-95,9$ \\
\hline
\end{tabular}

Fonte: Elaboração própria.

Nota: Sinal convencional utilizado:

- Dado numérico igual a zero não resultante de arredondamento.

Observa-se, na Tabela 2, a caracterização das violências financeira e sexual de acordo com os dados do agressor e da ocorrência. Os casos de violência financeira apresentaram como principal perpetrador indivíduos com idade até 59 anos, do sexo masculino; a maioria era conhecida das vítimas. Em mais da metade dos casos de violência, suspeitava-se do uso do álcool no momento da agressão. 
Tabela 2 - Caracterização das violências financeira e sexual praticadas contra a pessoa idosa, de acordo com os dados do agressor. Espírito Santo, Brasil - 2011-2018

\begin{tabular}{|c|c|c|c|c|c|c|}
\hline \multirow{2}{*}{ Variáveis } & \multicolumn{3}{|c|}{$\begin{array}{l}\text { Violência Financeira } \\
\qquad n=46\end{array}$} & \multicolumn{3}{|c|}{$\begin{array}{c}\text { Violência Sexual } \\
\qquad n=11\end{array}$} \\
\hline & $\mathbf{n}$ & $\%$ & $\begin{array}{c}\text { Intervalo de } \\
\text { Confiança 95\% }\end{array}$ & $\mathbf{n}$ & $\%$ & $\begin{array}{c}\text { Intervalo de } \\
\text { Confiança 95\% }\end{array}$ \\
\hline \multicolumn{7}{|c|}{ Idade do agressor (anos) } \\
\hline 0 a 59 anos & 35 & 94,6 & $79,8-98,7$ & 4 & 50,0 & $17,9-82,1$ \\
\hline 60 ou mais & 2 & 5,4 & $1,3-20,1$ & 4 & 50,0 & $17,9-82,1$ \\
\hline \multicolumn{7}{|l|}{ Sexo do agressor } \\
\hline Masculino & 27 & 60,0 & $44,7-73,5$ & 11 & 100,0 & - \\
\hline Feminino & 9 & 20,0 & $10,5-34,7$ & - & - & - \\
\hline Ambos & 9 & 20,0 & $10,5-34,7$ & - & - & - \\
\hline \multicolumn{7}{|c|}{ Vínculo com a vítima } \\
\hline Conhecido & 40 & 93,0 & $79,8-97,8$ & 9 & 81,8 & $46,5-95,9$ \\
\hline Desconhecido & 3 & 7,0 & $2,2-20,2$ & 2 & 18,2 & $4,1-53,5$ \\
\hline \multicolumn{7}{|c|}{ Suspeita de uso de álcool } \\
\hline Sim & 16 & 53,3 & $34,9-70,9$ & 2 & 33,3 & $6,8-77,4$ \\
\hline Não & 14 & 46,7 & $29,1-65,1$ & 4 & 66,7 & $22,6-93,2$ \\
\hline
\end{tabular}

Fonte: Elaboração própria.

Nota: Sinal convencional utilizado:

- Dado numérico igual a zero não resultante de arredondamento

Observa-se que a maioria dos casos de violência contra a pessoa idosa teve apenas um agressor. Quanto à ocorrência do abuso financeiro, em mais de 90,0\% dos casos ocorreu na residência, em zona urbana e já havia ocorrido anteriormente. Quanto ao turno, nota-se que grande parte aconteceu durante manhã ou tarde e na zona urbana. Além disso, a maioria dos casos foi encaminhada. Em relação à violência sexual notificada, metade dos perpetradores tinha até 59 anos e a outra metade 60 ou mais, todos pertenciam ao sexo masculino e eram conhecidos das vítimas. Na maioria dos casos não havia suspeita de uso de álcool e havia apenas um agressor. Todas as agressões ocorreram na residência, sendo grande parte à noite ou de madrugada. Vale destacar a repetição dos casos de violência e a zona urbana como de maior ocorrência. Todas as vítimas receberam encaminhamento (Tabela 3).

Tabela 3 - Caracterização das violências financeira e sexual praticadas contra a pessoa idosa, de acordo com os dados da ocorrência. Espírito Santo, Brasil - 2011-2018

\begin{tabular}{|c|c|c|c|c|c|c|}
\hline \multirow{2}{*}{ Variáveis } & \multicolumn{3}{|c|}{$\begin{array}{l}\text { Violência Financeira } \\
\qquad \mathrm{n}=46\end{array}$} & \multicolumn{3}{|c|}{$\begin{array}{c}\text { Violência Sexual } \\
\mathbf{n}=\mathbf{1 1}\end{array}$} \\
\hline & $\mathbf{n}$ & $\%$ & $\begin{array}{c}\text { Intervalo de } \\
\text { Confiança 95\% } \\
\end{array}$ & $\mathbf{n}$ & $\%$ & $\begin{array}{c}\text { Intervalo de } \\
\text { Confiança } 95 \%\end{array}$ \\
\hline \multicolumn{7}{|c|}{ Número de envolvidos } \\
\hline $\mathrm{Um}$ & 30 & 65,2 & $50,0-77,8$ & 9 & 81,8 & $46,5-95,9$ \\
\hline Dois ou mais & 16 & 34,8 & $22,2-49,9$ & 2 & 18,2 & $4,1-53,4$ \\
\hline \multicolumn{7}{|c|}{ Ocorreu na residência } \\
\hline Sim & 42 & 95,4 & $82,9-98,9$ & 11 & 100,0 & - \\
\hline Não & 2 & 4,6 & $1,1-17,1$ & - & - & - \\
\hline \multicolumn{7}{|l|}{ Turno de ocorrência } \\
\hline Manhã/Tarde & 17 & 80,9 & $56,9-93,2$ & 1 & 16,7 & $1,7-70,2$ \\
\hline Noite/Madrugada & 4 & 19,1 & $6,8-43,1$ & 5 & 83,3 & $29,8-98,3$ \\
\hline \multicolumn{7}{|l|}{ Violência de repetição } \\
\hline Sim & 40 & 93,0 & $79,8-97,8$ & 7 & 70,0 & $35,1-90,9$ \\
\hline Não & 3 & 7,0 & $2,2-20,2$ & 3 & 30,0 & $9,0-64,8$ \\
\hline
\end{tabular}


Tabela 3 - Caracterização das violências financeira e sexual praticadas contra a pessoa idosa, de acordo com os dados da ocorrência. Espírito Santo, Brasil - 2011-2018

\begin{tabular}{l|c|c|c|c|c|c}
\hline \multirow{2}{*}{ Variáveis } & \multicolumn{3}{|c|}{$\begin{array}{c}\text { Violência Financeira } \\
\mathbf{n = 4 6}\end{array}$} & \multicolumn{3}{c}{$\begin{array}{c}\text { Violência Sexual } \\
\mathbf{n}=\mathbf{1 1}\end{array}$} \\
\cline { 2 - 7 } & $\mathbf{n}$ & $\mathbf{0}$ & $\begin{array}{c}\text { Intervalo de } \\
\text { Confiança 95\% }\end{array}$ & $\mathbf{n}$ & $\mathbf{\%}$ & $\begin{array}{c}\text { Intervalo de } \\
\text { Confiança 95\% }\end{array}$ \\
\hline $\begin{array}{l}\text { Zona de ocorrência } \\
\quad \text { Urbana }\end{array}$ & 44 & 95,6 & $83,6-99,0$ & 8 & 80,0 & $42,9-95,5$ \\
$\quad \begin{array}{l}\text { Rural } \\
\text { Encaminhamentos }\end{array}$ & 2 & 4,4 & $1,0-16,4$ & 2 & 20,0 & $4,5-57,0$ \\
$\quad$ Sim & & & & & & - \\
$\quad$ Não & 34 & 77,3 & $62,1-87,6$ & 10 & 100,0 & - \\
\hline
\end{tabular}

Fonte: Elaboração própria.

Nota: Sinal convencional utilizado:

- Dado numérico igual a zero não resultante de arredondamento.

\section{Discussão}

O presente estudo evidenciou uma prevalência de 2,8\% de violência financeira e de 0,7\% de violência sexual contra a pessoa idosa. Estudo $^{(8)}$ encontrou prevalência de 0,7\% para violência sexual nos anos de 2011 e 2012. Estudo realizado em Florianópolis ${ }^{(9)}$ obteve a prevalência de 2,5\% de violência financeira, corroborando os achados desta pesquisa. A baixa prevalência das notificações desses tipos de violência contra os idosos no período estudado pode ser explicada por fatores como o seu não reconhecimento pela sociedade e a subnotificação ${ }^{(10)}$.

Apesar de a violência ser um agravo de notificação compulsória desde 2011 e o Estatuto do Idoso prever a comunicação de maus-tratos contra os idosos, o reconhecimento da violência contra essas pessoas enfrenta dificuldades políticas, sociais e culturais, que abrangem a identificação desses indivíduos como portadores de direitos e de autonomia para tomar suas próprias decisões ${ }^{(11)}$. Essas atitudes contribuem para que a violência contra a população idosa continue velada e invisível para famílias e sociedade. Pode-se considerar que a violência contra a pessoa idosa é expressão das relações entre os grupos etários que colocam o idoso como um ser dependente, suscetível e descartável ${ }^{(8,12)}$, influenciando na qualidade de vida e na morbimortalidade dessa população.
Outro fator que contribui para o silêncio ao redor da violência contra o idoso é a dificuldade das próprias vítimas em denunciarem esse tipo de situação. Isso ocorre porque, muitas vezes, eles têm medo de ser abandonados e de cortar vínculos afetivos com seus agressores ${ }^{(11)}$, o que indica que também a falta de suporte social é um fator de risco para a ocorrência de violência nessa população ${ }^{(13)}$. Essas dificuldades demonstram a necessidade de formação de uma rede de proteção que incluam também ações de fortalecimento da família e de apoio na prestação de cuidados aos idosos ${ }^{(14)}$.

A falta de notificação pode denunciar também a falha do sistema de defesa e proteção, contribuindo para a ocorrência silenciosa desses $\operatorname{agravos}^{(8,11)}$. Concorrem também para a subnotificação o despreparo dos profissionais de saúde para identificação, enfrentamento e acompanhamento dos casos aliado ao desconhecimento desses quanto à rede de atendimento e às políticas de proteção a essa população ${ }^{(10,15)}$.

Quando se trata das vítimas de violência financeira, é importante destacar o maior número de pessoas com idade mais avançada (70 anos ou mais), pertencentes ao sexo feminino e com companheiro, semelhante ao encontrado na literatura $^{(8,16)}$. Pesquisa por Amostra de Domicílios realizada em Minas Gerais, em 2014, demonstrou que a maioria dos idosos $(88,7 \%)$ era principal responsável pela renda familiar. Este fator, 
aliado à maior limitação funcional e cognitiva em idosos mais velhos ${ }^{(17)}$, pode explicar a maior frequência do agravo nessa faixa etária. Além disso, a maior expectativa de vida em mulheres do que em homens faz com que as idosas, por serem em maior frequência longevas ${ }^{(7)}$, possivelmente estejam mais expostas à violência. Também as questões de gênero perpassam a vida das mulheres, colocando-as em situação de maior vulnerabilidade quando idosas ${ }^{(12,17)}$.

Quanto à violência sexual, percebe-se, dentre as principais vítimas, mulheres de 60 a 69 anos, semelhante ao encontrado em estudo ${ }^{(12)}$ que identificou o abuso sexual como mais frequente entre as mulheres. Além disso, os resultados deste estudo apontaram maior proporção de casos de idosas que não possuíam parceiro íntimo, achado que vai ao encontro de pesquisa realizada nos Estados Unidos, onde as chances de idosas sofrerem violência sexual era duas vezes maior entre as que não eram casadas ${ }^{(18)}$. Vale ponderar ainda que a experiência de abuso sexual acarreta consequências na vida da mulher, que podem incluir desde o risco aumentado de infecção pelo HIV (vírus da imunodeficiência humana) até depressão e suicídio ${ }^{(19)}$.

Nesse contexto, ressalta-se que a residência da vítima, em ambos os casos, foi o principal local da perpetração da violência, assim como o principal agressor foi alguém conhecido, principalmente homens, como apontado por outros estudos $^{(7,15)}$. As questões de gênero são pouco levadas em consideração nessa fase da vida, todavia a discriminação de gênero ocorre do nascimento à velhice, em todas as classes sociais ${ }^{(20)}$.

As relações de poder do homem sobre a mulher iniciam-se na infância, quando os meninos são estimulados a serem dominadores e competitivos, enquanto às meninas cabe o papel de cuidadoras e empáticas. Dessa forma, o estereótipo de gênero torna-se enraizado, levando a uma assimetria de poder nas relações entre homens e mulheres, que perpassa por todas as idades ${ }^{(20)}$.

Em relação à escolaridade das vítimas, nota-se, em ambos os tipos de violência, que os idosos encontram-se no grupo de menor escolaridade.
Estes podem expressar maior dependência em atividades cotidianas ou mesmo financeiras, $\mathrm{O}$ que pode impor relações de poder entre o idoso e o cuidador ${ }^{(21)}$.

Outra característica que esteve presente nas notificações de violência foi a maior frequência de vitimados da cor preta ou parda. No Brasil, pessoas negras estão nas camadas sociais mais pobres, o que gera uma crônica situação de desigualdade, agravada pelo racismo. Este é responsável pelas diversas formas de discriminação presentes no cotidiano dessas pessoas, o que justifica o fato de essa população vivenciar mais esse agravo ${ }^{(22)}$.

De acordo com os resultados desta pesquisa, dentre os casos notificados de violência financeira e sexual observou-se menor proporção de pessoas com deficiências/transtornos. É importante ressaltar a limitação desses idosos, além da dependência em relação aos seus cuidadores até mesmo para acessarem os serviços de saúde e realizarem denúncias. Estudo ${ }^{(23)}$ identificou que a violência e a discriminação são mais frequentes em pessoas que têm algum tipo de deficiência ou transtorno.

Diferentemente do encontrado em outro estudo $^{(8)}$, a violência financeira contra o idoso foi de repetição. O fato de os idosos sentirem-se incapazes de gerenciar sua própria vida financeira e a dependência dos abusadores são possíveis causas para a continuidade desse tipo de agressão ${ }^{(12,15)}$. Além disso, ocorre também o medo da própria vítima de continuar sofrendo abusos ou ser abandonada e afastada do convívio familiar, caso resolva fazer a denúncia ${ }^{(8-9,15)}$.

O abuso de bebidas alcoólicas pelo agressor é um dos fatores de risco para a violência descritos pela literatura ${ }^{(8,12)}$. Estudo ${ }^{(10)}$ identificou uma chance 3,8 vezes maior de ocorrência de maus-tratos em idosos que tinham cuidadores que apresentavam problemas com álcool. No presente estudo foi encontrada maior prevalência de suspeita de uso de álcool somente na violência financeira, o que não foi encontrado para a violência sexual.

Observa-se, em relação aos encaminhamentos, que todos os idosos que foram vítimas 
de violência sexual receberam algum encaminhamento, porém o mesmo não foi percebido para os casos de idosos vítimas de violência financeira, havendo um quantitativo importante daqueles que não foram encaminhados, demonstrando a necessidade de avançar na rede de proteção a essas pessoas ${ }^{(24)}$. O atendimento completo às vítimas é essencial para a resolutividade dos casos e o rompimento do ciclo de violência, avançando, assim, na real garantia dos direitos dessas pessoas ${ }^{(15)}$. A zona de residência também é um fator limitante no acesso à rede de serviços de saúde ${ }^{(25)}$, o que pode ser inferido pelo menor número de notificações de agressão no meio rural.

Por fim, destaca-se o importante papel do setor saúde na prevenção e no combate à violência contra a pessoa idosa, além do atendimento e acompanhamento das vítimas e de suas famílias. Os profissionais de saúde estão em uma posição estratégica para a identificação de casos e dos fatores de risco para a ocorrência de violência, já que, muitas vezes, são o primeiro contato das vítimas. Estudo ${ }^{(16)}$ defende que toda ida do idoso a uma unidade de saúde constitui-se em oportunidade para a investigação de situações de violência, destacando-se o papel do profissional de enfermagem nesse processo e a necessidade de uma escuta atenta e qualificada.

Como limitação do presente estudo destaca-se o baixo número de publicações específicas sobre violência financeira e sexual contra os idosos, limitando a discussão dos resultados desta pesquisa. Nesse mesmo sentido, outro fator limitador são as poucas notificações desses agravos, devido às dificuldades dos idosos em denunciar as ocorrências ${ }^{(8,12,15)}$, bem como dos profissionais de saúde em notificá-las.

\section{Conclusão}

O presente estudo permitiu concluir-se que as violências financeira e sexual são pouco notificadas, todavia as prevalências encontradas são similares ao revelado pela literatura. Quanto às características das vítimas, em ambos os abusos estudados, as pessoas idosas são do sexo feminino, com baixa escolaridade, de raça preta ou parda e sem deficiência/transtorno. As agressões, em sua maioria, foram cometidas por uma única pessoa, do sexo masculino, conhecido da vítima, praticadas no domicílio e com histórico de repetição.

Importante destacar que idosos vitimados pela violência financeira, em sua maioria, apresentavam companheiros, o agressor tinha suspeita de abuso de álcool, e a violência acontecia durante o dia, enquanto a violência sexual foi mais frequente em idosos sem companheiros e a ocorrência se dava durante a noite/madrugada.

Os resultados da pesquisa são de grande importância para os profissionais de saúde, em especial o enfermeiro, pois evidencia a vulnerabilidade da pessoa idosa à experiência de violência. Nesse sentido, é fundamental que o enfermeiro, durante os atendimentos, investigue situações de violência vivenciadas por essa população, e notifique esse agravo de modo a romper com o ciclo de violência e promover um cuidado integral e qualificado. Vale reforçar que o processo de notificação faz parte da atuação do profissional de saúde, sendo importante que tais profissionais estejam qualificados para tal procedimento, uma vez que os dados da notificação são de grande relevância para a elaboração de políticas preventivas e de enfrentamento da violência da população idosa.

\section{Colaborações:}

1 - concepção, projeto, análise e interpretação dos dados: Franciele Marabotti Costa Leite e Gracielle Pampolim;

2 - redação do artigo e revisão crítica relevante do conteúdo intelectual: Franciele Marabotti Costa Leite, Gracielle Pampolim, Mayara Alves Luis, Raniele de Paula Silva e Márcia Regina de Oliveira Pedroso;

3 - aprovação final da versão a ser publicada: Franciele Marabotti Costa Leite e Gracielle Pampolim. 


\section{Referências}

1. Mendes ACG, Sá DA, Miranda GMD, Lyra TM, Tavares RAW. Assistência pública de saúde no contexto da transição demográfica brasileira: exigências atuais e futuras. Cad Saúde Pública. 2012 May;28(5):955-64. DOI: http://dx.doi. org/10.1590/S0102-311X2012000500014

2. Minayo MC. Violence against the elderly: the relevance of an old health problem. Cad Saúde Publica. 2003;19(3):783-91. DOI: http://dx.doi. org/10.1590/S0102-311X2003000300010

3. World Health Organization. Elder Abuse: The Health Sector Role in Prevention and Response [Internet]. Geneva; 2016 [cited 2019 Aug 28]. Available from: https://www.who.int/violence_ injury_prevention/violence/elder_abuse/WHO_ EA_ENGLISH_2017-06-13.pdf?ua=1

4. Pillemer K, Burnes D, Riffin C, Lachs MS. Elder Abuse: Global Situation, Risk Factors, and Prevention Strategies. Gerontologist. 2016;56(Suppl2):194-205. DOI: http://dx.doi.org/10.1093/geront/gnw004

5. Bond MC, Butler KH. Elder abuse and neglect: definitions, epidemiology, and approaches to emergency department screening. Clin Geriatr Med. 2013 Feb;29(1):257-73. DOI: https://doi. org/10.1016/j.cger.2012.09.004

6. Brasil. Presidência da República. Secretaria de Direitos Humanos. Manual de enfrentamento à violência contra a pessoa idosa: é possível prevenir, é necessário superar. Brasília (DF); 2014.

7. Lopes LGF, Leal MCC, Souza EF, Silva SZR, Guimarães NNA, Silva LSR. Violência contra a pessoa idosa. Rev Enferm UFPE. 2018;12(9):225768. DOI: https://doi.org/10.5205/1981-8963v12i9a236609p1129-1139-2018

8. Rocha RC, Côrtes MCJW, Dias EC, Gontijo ED. Violência velada e revelada contra idosos em Minas Gerais-Brasil: análise de denúncias e notificações. Rev Saúde debate. 2018;42(spe4):81-94. DOI: http://dx.doi.org/10.1590/0103-11042018s406

9. Bolsoni CC, Coelho EBS, Giehl MWC, D’Orsi E. Prevalence of violence against the elderly and associated factors-a population based study in Florianópolis, Santa Catarina. Rev Bras Geriatr Gerontol. 2016;19(4):671-82. DOI: http://dx.doi. org/10.1590/1809-98232016019.150184

10. Lino VTS, Rodrigues NCP, Lima IS, Athie S, Souza ER. Prevalence and factors associated with caregiver abuse of elderly dependents: The hidden face of family violence. Ciênc saúde coletiva. 2019 Jan;24(1):87-96. DOI: http://dx.doi. org/10.1590/1413-81232018241.34872016

11. Irigaray TQ, Esteves CS, Pacheco JTB, Grassi-Oliveira R, Argimon IIL. Maus-tratos contra idosos em Porto Alegre, Rio Grande do Sul: um estudo documental. Estud Psicol (Campinas). 2016;33(3):543-51. DOI: http://dx.doi. org/10.1590/1982-02752016000300017

12. Mascarenhas MDM, Neves ACM, Silva MMA, Malta DC, Andrade SSCA, Pedrosa AAG. Violence against the elderly: Analysis of the reports made in the health sector - Brazil, 2010. Ciênc saúde coletiva. 2012;17(9):2331-41. DOI: http:// dx.doi.org/10.1590/S1413-81232012000900014

13. Johannesen M, LoGiudice D. Elder abuse: a systematic review of risk factors in communitydwelling elders. Age Ageing. 2013;42(3):292-8. DOI: http://dx.doi.org/10.1093/ageing/afs195

14. Cachina AMP, Lemos de Paiva I, Torres TL. Violência intrafamiliar contra idosos: revisão sistemática. Liber. 2016 Jul;22(2):185-96.

15. Rodrigues RAP, Santos AMR, Pontes MLF, Monteiro EA, Fhon JRS, Bolina AF, et al. Report of multiple abuse against older adults in three Brazilian cities. PLoS ONE. 2019;14(2):e0211806. DOI: https://doi.org/10.1371/journal.pone.0211806

16. Silva GCN, Almeida VL, Brito TRP, Godinho MLC, Nogueira DA, Chini LT. Violência contra idosos: umaanálisedocumental. quichan.2018;18(4):449-60. DOI: http://dx.doi.org/10.5294/aqui.2018.18.4.7

17. Duque AM, Leal MCC, Marques APO, Eskinazi FMV, Duque AM. Violência contra idosos no ambiente doméstico: prevalência e fatores associados (Recife/ PE). Ciênc saúde coletiva. 2012;17(8):2199-208. DOI:http://dx.doi.org/10.1590/S1413-81232012000800030

18. Cannell MB, Manini T, Spence-Almaguer E, Maldonado-Molina M, Andresen EM. U.S. Population Estimates and Correlates of Sexual Abuse of Community-Dwelling Older Adults. J Elder Abuse Negl. 2014;26(4):398-413. DOI: https://doi.org/10.1080/08946566.2013.879845

19. García-Moreno C, Jansen HA, Ellsberg M, Heise L, Watts $\mathrm{CH}$. WHO multi-country study on women's health and domestic violence against women: initial results on prevalence, health outcomes and women's responses. Genebra: WHO; 2005.

20. Cross C, Campbell A. Violence and Agression in Women. In: Shackelford T, Hansen R. The 
Evolution of Violence. New York: Springer; 2013. p. 211-32.

21. Aguiar MPC, Leite HA, Dias IM, Mattos MCT, Lima WR. Violência contra idosos: descrição de casos no Município de Aracaju, Sergipe, Brasil. Rev Esc Anna Nery. 2015;19(2):343-9. DOI: http:// dx.doi.org/10.5935/1414-8145.20150047

22. Brasil. Ministério da Saúde. Secretaria de Gestão Estratégica e Participativa. Painel de Indicadores do SUS n. 5: prevenção de violências e cultura de paz. Brasília (DF); 2008.

23. Dammeyer J, Chapman M. A national survey on violence and discrimination among people with disabilities. Rev BMC Public Health. 2018;18(355). DOI: https://doi.org/10.1186/s12889-018-5277-0
24. Plassa BO, Alarcon MFS, Damaceno DG, Sponchiado VBY, Braccialli LAD, Silva JAVE, et al. Fluxograma descritor no atendimento à pessoa idosa vítima de violência: uma perspectiva interdisciplinar. Esc Anna Nery. 2018;22(4):e20180021. DOI: https://doi.org/10.1590/ 2177-9465-EAN-2018-0021

25. Kassouf AL. Acesso aos serviços de saúde nas áreas urbana e rural do Brasil. Rev Econ Sociol Rural. 2005;43(1):29-44. DOI: http://dx.doi.org/10.1590/ S0103-20032005000100002

Recebido: 29 de agosto de 2019 Aprovado: 12 de novembro de 2019

Publicado: 17 de fevereiro de 2020

A Revista Baiana de Enfermagem utiliza a Licença Creative Commons - Atribuição-NãoComercial 4.0 Internacional.

https://creativecommons.org/licenses/by-nc/4.0/

Este artigo é de acesso aberto distribuído sob os termos da Licença Creative Commons (CC BY-NC). Esta licença permite que outros remixem, adaptem e criem a partir do seu trabalho para fins não comerciais. Embora os novos trabalhos tenham de lhe atribuir o devido crédito e não possam ser usados para fins comerciais, os usuários não têm de licenciar esses trabalhos derivados sob os mesmos termos. 